(2) Facts relating to the duration of zentricular systole and diastole. Without cardiography there are no means of determining, even approximately, the duration of the periods of contraction and relaxation of the ventricle; with cardiography these durations are determined with the greatest facility and accuracy. The cardiogram, as stated, marks the beginning and end of these phases, and determination of their duration is only a matter of measurement on a horizontal line. Concerning these points investigation has brought out the following formula of results :

A.-With pulse-rate at 75 per minute, (cycle fourfifths $=.800$ second), the average duration of systole is about .326 second, and of diastole about .474 second.

B.-In the small variations of cyclic rythm systole usually also varies, but its variations may be either with or against the sense of the variations of the cycles. In considerable cyclic variation systole is shorter with short, and longer with long cycles.

C.- In the small variations of cyclic rhythm diastole usually, also, varies, and in much greater degree than systole, and almost always in the same sense as the cyclic changes. In considerable cyclic change the diastolic shortening or lengthening is correspondingly large.

D.- Systole and diastole may both vary their duration without change of the cycles, in which case the shortening of one will exactly equal the lengthening of the other.

In illustration of these statements figure 5 may also be studied. The systoles were carefully measured and the diastoles computed by deducting the latter from the corresponding cycles. The figures within the divisions express, in decimals of a second, the values found. The pulse-rate of the individual cycles of the run varies between 80 and 85 per minute, and the average rate of the nine cycles is $8 \mathrm{I} .5$ per minute.

(3). Facts relating to auricle. - Although the theory, that auricular contraction occurred in the latter part of the period of ventricular relaxation, was well accepted, it remained for cardiography to produce indubitable demonstration of the fact, and definitely determine the relative position and time of this event. Graphic experiments on animals and on man go to prove that the systole of the auricle precedes the systole of the ventricle by an interval which averages about one-fifth the duration of the cardiac cycle; the proportion, however, being less in long, and greater in short cycles. In animals the auricular trace, as obtained directly from the auricle, is in form of a pronounced single wave; but in man the trace, as obtained indirectly through the ventricle, is in form of two small waves, as said before. The double wave of man occupies the same proportional interval as the single wave of animals.

These are specimens of the results of pure cardiography. In a word, by it we learn the form and chronometry of the heart's pulsations. The faets, if not extensive in range, are such as may be multiplied by every experimentation, and like those of clinical thermometry become innumerable. They commend themselves for their positiveness and pre- cision, and as supplying a void in our knowledge of the finer physiology of the heart's movements. Besides, they claim our appreciation in that they are essential and preparatory to the richer and more practical developments of simultaneous cardiosphygmography.

\section{SYMPATHETIC OPHTHALMIA WITH OSSIFIC AND CALCAREOUS METAMORPHOSIS. ${ }^{1}$}

\section{BY FLAVEL, B. TIFFANV, M.D.,}

\author{
OF KANSAS CITY, MO.
}

It is with some hesitancy that I come before this assembly with a paper on sympathetic ophthalmia, a subject the literature of which is so voluminous and opinions so greatly at variance. But considering the importance of the subject, the frequency of its occurrence and its direful results, it may not be out of place for me to report some cases which have come under my observation. The cases which I shall present are complicated with some ossific or calcareous metamophoses; one having a worm, lanceolatum distoma, of the choroid.

Ever since sympathetic ophahalmia was first announced by Mackenzie, the disease has been looked upon as one of the most dangerous and formidable which the eye is heir to. Its approach is insidious, and when the flame is once kindled, it is extremely difficult to smother it before great damage is done. Plastic exudations have taken place ere the patient is aware that he has any difficulty of the eye; adhesions are formed, binding the iris to the anterior capsule of the lens and the pupilary area is filled or the pupil is occluded and blindness ensues. That sympathetic ophthalmia is a plastic inflammation of some portion of or of the entire uveal tract, is quite generally acknowledged. In considering the pathology of the disease, it will, no doubt, be agreed that it is a disease peculiar to the uveal tract, and that the ciliary body and parts in near proximity are the first to be involved. But when we revert to the etiology of the disease, I am sure that there will be a difference of opinion as to whether it is a transmitted or a reflected affection. According to my observations and data collected. I must regard the affection as a reflected disease through the medium of the ciliary nerves, notwithstanding Arlt's strong argument in favor of its being a continuation of an inflammation of the optic nerve through the optic commissure; or the assertions of others who claim to have traced the hypothetical microbe of the offending member through the optic nerve to the other eye. In support of the theory that sympathetic ophthalmia is a reflected disease and that it is through the medium of the ciliary nerves, and due to diseased filaments of these nerves, and not a progressive inflammation through the optic nerve, I give the following data:

I. Sympathetic ophthalmia is a plastic inflammation, a form of inflammation peculiar to vascular tissue not to nerve tissue.

2. It is an inflammation of the uveal tract usually

IRead in the Section of Ophthalmology and Otology at the ThirtySixth Annual Meeting of the American Medical Association. 
of the ciliary body, frequently involving the iris and choroid while the retina remains intact.

3. In inflammation of nerve tissue it is the neurilemma or medullary portion rather than the axial part that is involved.

4. 'The optic nerve sheath does not enter the eye but passes over on to the sclera, leaving the nerve to enter the eye as a non-medullary nerve.

5. The ciliary nerves enter the eye and do not leave their medullary portion until they are lodged in the ciliary region.

6. The ciliary nerves go to supply, and are intimately connected with the uveal tract, and ciliary region, whereas the optic nerve has no immediate connection with this tract.

7. Were the optic nerve the medium of transmis sion or propagation of the inflammation by extension, enucleation would not be likely to arrest the inflammation.

8. Sympathetic ophthalmia occurs in eyes with completely atrophied, and even cretaceous degeneration of the optic nerve.

9. The sympathetic irritation has been arrested by division of the ciliary nerves near their entrance into the sclera, leaving the optic nerve intact.

The cases which I wish to present, are interesting and instructive, both as corroborating what has been said regarding the etiology of the disease, and in the specific phenomena of calcareous and ossific degeneration.

Case 1.-Charlotte K., a Swede, aged 64, was led into my eye and ear clinic on Sept. 30, r884. With her right eye she could but count figures 16 inches away, while she was totally blind in the left. At I $5_{5}$ years of age, while playing on the grass one day, she accidentally discovered that she was blind in the left eye. She asked her mother if it was true with other people as it was with her, that if they covered the right eye they could not see; whereas if only the left eye was covered the sight was good? 'This was the first knowledge her parents or friends had of her blindness. She was taken to a physician in Sweden, who told the parents that their daughter had a gray star of the left eye. There was no remembrance of her ever having received an injury or of her having had an affection of any kind in the eye, nor had she ever had any pain or soreness there; surgical or medical treatment of course she had not had. "The sight of her right eye had always been good up to a few weeks previous to her first visit to me. Up to that time she had never experienced inconvenience of any kind from this eye. At this time, Sept. I5, I 884 , when milking, the cow switched her tail in the patient's face, striking her left eye with some force, occasioning considerable pain which subsided after a few hours; three or four days subsequently her right eye began to grow dim, the dimness gradually increased up to the time when I first saw her, when, as before stated, she had only a very small amount of vision.

Ophthalmoscopic examination revealed choroiditispigmentosa, floating bodies in the vitrious, with some slight opacity of the lens. Diagnosis: Choroiditis with incipient neuclear cataract of $R$. E. 'There was a very dense leucoma of the left, which would not permit an examination of any of the internal structures. 'The cornea also had centres of cretaceous metamorphoses; the intra-ocular tension was considerably increased especially at the posterior portion where the eye firmly resisted any pressure. I regarded the eye as an old glaucomatous eye, and the source of the irritation to its fellow; and accordingly urged immediate enucleation, notwithstanding there was no pain or soreness, nor ever had been except for the short interval succeeding the injury from the cow's tail.

As I enucleated the eye, it was accidently dropped and fell to the floor, giving a sound like that of a stone or bone. Upon making a transverse section, the following macroscopical conditions were found: Cornea hypertrophied to several times its normal thickness, entirely leucomatous with extensive calcareous metamorphoses. The crystalline lens was quite calcified in situ; the anterior chamber obliterated and iris in juxtaposition with the cornea; the retina completely detached, and coarcted by an osseous cup, which occupied the whole fundus of the eye (with the exception of that part opposite the optic nerve) and extending in part to the ora-serrata. The opening at the optic nerve, through which passes the retina, measures a fourth of an inch in diameter, and at this part the plate or cup of bone is at least a fourth of an inch in thickness, while it gradually decreases in thickness as it approaches the ora-serrata. In I. Kn. Knapp's able article on "Bone Formation in the Choroid," he asserts that these ossific deposits of the eye are only found in the choroid, and that they invariably begin in the inner or capillary layer.

In making sections of different parts of the choroid, I find that this case bears him out in the assertion that the ossification begins in the capillaries, but, instead of its being near the ora-serrata or ciliary body in the case under consideration, the ossification evidently began near the entrance of the posterior ciliary nerves and arteries, where the cup is the thickest. The osseous formation seems to be wholly confined to the inner layer of the choroid, involving more particularly the radicals or capillaries.

Dr. Alfred Voorhies speaks of a bony formation of the crystalline lens (no ossific deposit elsewhere). He claims to have found in his specimen Harvasion canals, canaliculæe, and lacunæ, but he does not attempt to show how the bone was developed, and in what way the canals and canalicula were formed. If the lens, or even the capsule, possessed bloodvessels, we might more easily account for the phenomenon; or, in other words, we can account for bone formation in vascular tissue, where there is constantly an interchange of fluid; but even here we do not expect to find the same structure as in the physiological formation of bone; for how could we have Haversian zones or lamellae canals, and canalicula, without, first, cartilaginous tissue with its cells surrounded by zones and covered by vascular perichondrium.

Turning to the microscopical examination of the case under consideration, we find that it illustrates 
forcibly the hypothesis that Dr. H. Knapp makes The portion of the lens which had not been absorbed, prominent in his article, that ossification always begins in the capillaries.

Sections of this specimen show canals, lacunæ, and osteoblasts, but the Haversian zones or lamellee do not appear. How could they without a previous cartilaginous skeleton to build upon? Another point of interest is the time of development of the osseous growth. From the fact that the patient was blind of this eye from the time that she was 15 years old (possibly from birth), and has now attained the age of 64 , the bone may have been been many years in forming. The bone formed all around the optic in nerve, crowding against it, even cleaving the retina from the choroid throughout its whole extent. Yet no sympathetic ophthalmia was produced until the bone had reached the ciliary region; a fact going so far as one case can, to prove the theory that sympathetic ophthalmia is a reflected, not a transmitted disease.

After enucleation this patient's vision was increased to ${ }^{20 / 70}$, and the symptoms of hyalitis passed away. I have not seen the patient for several months, and do not know the condition of the cataract

Cise 2.- Kate B., aged 2o, visited me May $2 \mathrm{I}$, i $88 \mathrm{r}$, for an affection of the right eye. The following is the history as given by herself: "When a : child in school I was troubled with pain in my eyes, especially in the right. The pain was also severe through the temples and over the brow; this pain was intensified by close application to study. One day I was kept after school to learn a lesson in geography, which I had failed to get on account of this severe pain; the lesson required close looking, being a map lesson. I told the teacher that my eyes pained me so that I could not study; but she insisted on my haying my lesson then and there. I went home that night with a terrible pain in my eye, which lasted for weeks. I was unable to attend school or read a word for two years, at the end of which time I attempted to resume my school work, but my eyes troubled me so that I was unable to attend regularly. I went to school, off and on, for two years longer, when I was obliged to desist from all study. In the winter that I was 16 my health was very poor, and the pain in my right eye was almost constant. My eyes were then crossed. Four years later an operation was made on my right eye for the cast. After this operation, the eye was much worse."

When Miss B. visited me, I diagnosticated secondary cataract in the right eye, with posterior synechae; and in the left, a high degree of hypermetropia. I made an iridectomy of the right, and corrected the hypermetropia of the left eye. In a few months I made keratonyxis of $R$. E. The eye did very well for a time, when repeated paroxysms of glaucomatous pain appeared, destroying entirely the sight. There were at this time symptoms of sympathetic ophthalmia in the left eye. Three years ago last Christmas I enucleated the right eye.

Upon cutting the globe open I found an ossific deposit in the choroid, embracing about one-fifth of this membrane, at the fundus near the macula lutea.

was calcified. After removing the eye there was considerable hamorrhage, which gave some trouble in controlling, and for which I tamponed the orbit with a sponge, leaving it in over night. The next day when I attempted to dress the orbit, I found to my surprise that sponge-grafting had taken place; so firmly did the sponge adhere to the stump, that I was obliged actually to tear it away, leaving some small bits in the wound. Ever since the removal of the eye the patient has suffered more or less pain in and about the orbit; abscesses would occasionally form in the conjunctiva of the stump, discharging into the orbit and through the nose. 'The periphery of the orbit has always been very tender. So great has been the hyperasthesia, that the patient has never been able to wear an artificial eye with any comfort. The pain and tenderness of the stump and surrounding parts have always been increased during the catamenia, and for the past year these periodic parox isms have assumed a much graver aspect than previously, the pain no longer being confined to the right side, but frequently attacking the left eye also, and culminating in severity at the occiput, or base of the brain. While the pain lasted the patient would complain of nausea, and of seeing a halo of prismatic colors when looking at the light of a lamp, great dimness of sight. In fact many of the symptoms of glaucoma were present.

Regarding these symptoms as pathognomonic of sympathetic ophthalmia, and looking for the cause in the cicatrix of the stump of the eye removed, I determined to excise the cicatrix: accordingly, Feb. 6,1885 , I resected a portion of the stump, since which time the patient has not suffered the least pain in the empty orbit, and although there have been now and then slight twinges of pain in the left eye, no paroxysm has occurred, nor has there been any dimming of sight. I think, therefore, we may conclude that the source of the irritation was located in the cicatrix of the stump, where, probably, there was entangled filaments of the ciliary nerves, upon whose liberation the symptoms of sympathetic ophthalmia immediately vanished.

Before concluding, I wish to call attention to another interesting specimen of ossific metamorphosis of the eye. In Dr. H. Knapp's article on "Bone Formation," he emphatically states that the choroid, or the uveal tract, is the only part of the eye which is susceptible to ossific metamorphosis. In this specimen, I think you will agree with me that there is at least an apparent bony formation in the sclera, embracing a rim nearly half an inch in width, in the region of the sclero-corneal junction, while the uveal tract is entirely free from the slightest ossific deposit.

In the fundus of this eye near the optic nerve we find a worm, about a half inch long, embedded in the choroid even to the sclera. I am sorry to say that I have no history of this eye, as it was not cut open at the time of enucleation, but placed in alcohol for future examination. 1 only know that it was diagnosed as a glaucomatous eye, and removed to check imminent sympathetic ophthalmia. 